\title{
EXPLORING THE IMPACT OF PERSONALITY TRAITS AND TECHNICAL AFFINITY ON THE APPEARANCE OF TECHNOSTRESS
}

\author{
Maximilian Lang and Christian Schieder \\ Ostbayerische Technische Hochschule Amberg-Weiden, Germany
}

\begin{abstract}
Information and communication technologies, such as instant messengers, have become an essential part of every person's work and private life. Undesirable side effects, such as technostress accompany this trend. The present study examines the relationship between the Big Five personality traits and the technical affinity to the appearance of technostress and its effect on the general task fulfillment. The experimental design's central component was an online memory game, combined with the NEO Five-Factor Inventory and a technical affinity questionnaire. The experiment with 13 participants was monitored with a gaze tracking device and corresponding software. This study showed that people with certain personality traits perceive technostress at a higher or lower level. Furthermore, technostress lowers task performance. However, this effect can be mitigated by a higher level of technical affinity. This paper is ongoing research. Therefore, future research should consider a higher number of participants and a variation of stressors.
\end{abstract}

\section{KEYWORDS}

Technostress, Personality Traits, Technical Affinity, Task Performance

\section{INTRODUCTION}

Digitization connects the entire world. Thanks to the increasing spread of information and communications technology (ICT), people are effortlessly connected anytime, anywhere. Of course, individuals, students, and employees alike benefit from this development through faster decision-making, smooth communication, and increased productivity. However, this trend is associated with adverse side effects, such as technology-induced stress, characterized as technostress. It is defined as "a modern adaptive disorder caused by the inability to use new computer technologies in a healthy way" (Brod, 1984).

Previous studies have focused primarily on the technostress creators' influence on a particular outcome (Sarabadani, Carter and Compeau, 2018; Benzari, Khedhaouria and Torrès, 2020). However, little is known about the relationship between the different personality traits and the emergence of technostress. The same applies to the influence of technical affinity on the occurrence of technostress. Therefore, this study aims to measure the influence of technostress on the overall task performance, based on the following research questions (RQ):

RQ1: "Which influence do personality traits have on the occurrence of technostress?

RQ2: "Which influence does technical affinity have on the occurrence of technostress?

RQ3: "Which influence does technostress have on the performance of tasks?

To answer these questions, a research model, including seven hypotheses, was developed. An experiment based on an online memory game was conducted to test the hypotheses. The personality traits of the participants were determined using the NEO Five-Factor Inventory. The attendees answered the questionnaire to determine their technical affinity. Also, eye-tracking software had monitored the entire experiment. The paper is structured as follows. First, the ethical explanation is mentioned, followed by the hypotheses and the research model. Then the analysis and the results are described. A discussion of the results follows this, and finally, a conclusion, the limitations, and future research are presented. 


\section{EXPERIMENT}

\subsection{Research Model and Hypotheses}

In order to answer the defined research questions, the following research model and hypotheses were made:

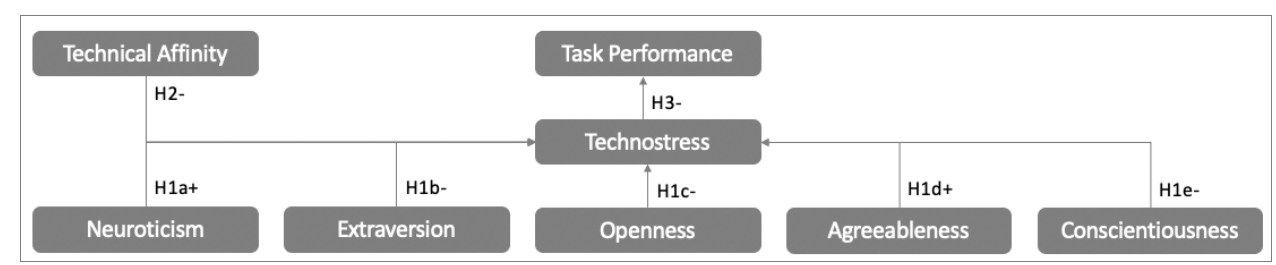

Figure 1. Research Model and Proposed Hypotheses

Neuroticism: According to Costa \& McCrae, individuals with this personality trait can be defined as "anxious, generally apprehensive, and prone to worry" (Costa and McCrae, 1992). Furthermore, these people are described as "being poor at controlling [their] impulses and desires, and [they are] unable to handle stress well" (Costa and McCrae, 1992). Based on the definition of neuroticism, the following hypothesis can be made up:

H1a: Neuroticism is positively related to the appearance of technostress.

Extraversion: An individual with a high level of extraversion can be described as "having a high level of energy and likes to keep active and busy" (Costa and McCrae, 1992). Devaraj et al. suggested that those persons tend to use technologies to maintain their social image. Hence, it is likely for them to perceive technostress as an opportunity rather than a threat (Devaraj, Easley and Michael Crant, 2008). Based on the definition of extraversion, the following hypothesis can be made up:

H1b: Extraversion is negatively related to the appearance of technostress.

Openness: These Individuals are open to new ideas and strive for new opportunities. In this way, highly valued people in the dimension of openness will have positive insights about technostress. These circumstances are seen as an opportunity to perform better (Chandra, Srivastava and Shirish, 2015). Based on the definition of openness, the following hypothesis can be made up:

H1c: Openness is negatively related to the appearance of technostress.

Agreeableness: Persons with a high level of agreeableness are "kind, considerate, likable, helpful and cooperative" (Graziano and Eisenberg, 1997). Due to their personality, they experience technostress as a threat (Srivastava, Chandra and Shirish, 2015) Based on the definition of agreeableness, the following hypothesis can be made up:

H1d: Agreeableness is positively related to the appearance of technostress.

Conscientiousness: Following Costa \& McCrae, these individuals are "reasonably efficient and generally sensible and rational in making decisions" (Costa and McCrae, 1992). Consequently, it is more likely for them to perceive technostress as a positive appearance. Based on the definition of conscientiousness, the following hypothesis can be made up:

H1e: Conscientiousness is negatively related to the appearance of technostress. 
Technical affinity: Is defined as a personal behavior that expresses itself in a positive attitude, enthusiasm, and trust towards technology (Karrer et al. 2009). It consorts with interest and acceptance of the technology. Technical affinity also has a positive effect on knowledge and experience with technology (Karrer et al. 2009). Based on the definition of technical affinity, the following hypothesis can be made up:

$\mathrm{H} 2$ : Technical affinity is negatively related to technostress.

Task Performance: According to Burton-Jones \& Straub, task performance is defined as the "extent to which an individual's task output is effective in meeting task objectives" (Burton-Jones and Straub, 2006). Moreover, interruptions can lead to a reduction in task performance (Tams, Thatcher and Grover, 2018). Based on the definition of task performance, the following hypothesis can be made up:

H3: Technostress is negatively related to task performance.

\subsection{Materials and Methods}

The experimental setting is designed as a true experiment. The participants should play an online memory game, followed by a survey referring to the technical affinity and the NEO-Five-Factor Inventory (NEO-FFI). Due to restrictions caused by the corona pandemic, the participants were selected using the convenience sampling method. 13 persons with german citizenship (six male, seven female) in the age group of 22-28 with a mean age of $M=25$ were selected randomly into two groups: The first group was defined as test group with $n=8$ (four male, four female), the second group as the control group with $n=5$ (two male, three female). A person can only be assigned to one of the two groups and participated in an order. The characteristics of the final sample are shown in Table 1.

Table 1. Sample Selection

\begin{tabular}{cccc}
\hline Measure & Items & Quantity & \% \\
\hline Age & $22-25$ & 9 & 69 \\
& $>25$ & 4 & 31 \\
\hline Test group & Male & 4 & 50 \\
& Female & 4 & 50 \\
\hline Control group & Male & 2 & 40 \\
& Female & 3 & 60 \\
\hline
\end{tabular}

The online memory consisted of $4 \times 8$ cards with cartoon-like images in different colors. The task was to find matching pairs of images by flipping two cards in each round. In the beginning, the cards were displayed face down. Whenever the participants identified matching pairs, the cards remained open. Otherwise, the cards were turned back to their original position (Tams, et al. 2018). The task was fulfilled when all 16 pairs were matched correctly. The number of moves and the time it took to complete the task was recorded to measure the task performance. The memory was selected due to easiness and understandability to cause as little stress as possible (Washburn et al. 2007).

The control group fulfilled the task without any interruptions. On the opposite, the test group was affected by upcoming instant messages on the screen's right bottom. These messages were sent in a pre-defined frequency (15 seconds) containing fun facts. Furthermore, the notifications were combined with a typical audio signal. All participants conducted the task in the same environment and setting to ensure a ceteris paribus condition.

An eye-tracking device has been used for both groups to receive a reliable set of physiological data. This instrument should detect whether and how often the test group was distracted by the upcoming notifications. First, the eye movement of the participants was calibrated by the system. They were asked to reduce their head movement during the experiment to a very minimum to ensure accurate measurement. A so-called area of interest (AOI) was defined to quantify the test group results. This area precisely reflects the area of the popup notifications. The measured time and count of views were the decisive variables to measure the appearance of technostress. 
To determine the influence of technical affinity a survey was conducted. Karrer et al. developed the chosen questionnaire (Karrer et al. 2009), which included 19 questions about the personal assessment of technical and electronic devices. For the measurement, a five-point Likert-Scale with the following statements ( $5=$ strongly agree, $4=$ agree, $3=$ neutral, $2=$ disagree, $1=$ strongly disagree) has been used. Negative statements were inverted. Consequently, the higher the mean-value, the higher the degree of technical affinity. The reliability and validity of the survey were proven. For validation, it was tested on a large heterogeneous sample. The survey includes several factors of technical affinity and has satisfactory quality criteria (Karrer et al. 2009).

The NEO-FFI by Costa \& McCrae was applied to identify the participants' personalities. The NEO-FFI (Costa and McCrae, 1992) was applied to investigate the five-factor model of personality. Consequently, a questionnaire containing 60 questions referring to the dimensions of the normal personality (neuroticism, extraversion, openness, agreeableness, and conscientiousness) was used (Schmitz et al. 2001). The survey is categorized into five dimensions, with 12 questions each. Again, a five-point Likert-Scale with the following statements $(5=$ strongly agree, $4=$ agree, $3=$ =neutral, $2=$ disagree, $1=$ strongly disagree) was deployed. It shows the quota sample representative of the population with T-values $(50+10 \mathrm{z})$. Table 2 shows the defined ranges of the participants' standard value.

Table 2. Categorization of NEO-FFI Results

\begin{tabular}{lcccc}
\hline Description & SD & Lower Limit & Upper Limit & SD \\
\hline Far below average & -3 SD & 20 & 30 & -2 SD \\
\hline Below average & -2 SD & 30 & 40 & - SD \\
\hline Average & - SD & 40 & 60 & + SD \\
\hline Above average & + SD & 60 & 70 & $+2 S D$ \\
\hline Far above average & $+2 S D$ & 70 & 80 & $+3 S D$ \\
\hline
\end{tabular}

The reliability and validity of the NEO-FFI were proven by a large sample and is one of the most applied tests to evaluate the five-factor model dimensions (Schmitz et al. 2001). The factors treatment and counterbalance were considered to increase the general validity of the experiment (Bhattacherjee, 2012). The experimental design aims to moderate different experience levels. Also, the duration was kept as short as possible to minimize boredom. There was no uncontrolled contact between the participants (Cassar, Friedman and Schneider, 2009).

All participants submitted a written declaration of consent following the General Data Protection Regulation (GDPR). After the experiment, the participants were informed about the study's purpose and the further data analysis process. All persons participated voluntarily and agreed to the use of their data for the research work.

\subsection{Results}

In the following chapter, the results of the experiment are analyzed based on the Research Question and the associated hypotheses. Figure 2 shows a summary of the results.

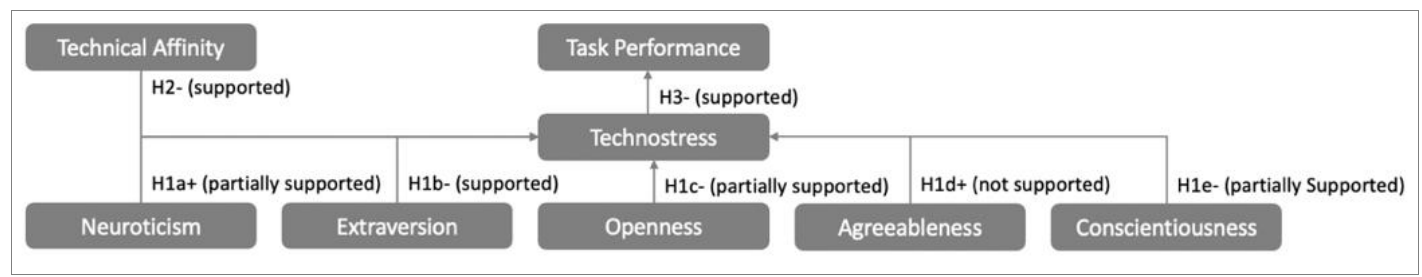

Figure 2. Summary of Results

\subsubsection{Relationship between Personality Traits and the Appearance of Technostress}

Neuroticism: The average time and the average number of views in the AOI were measured to test H1a. Three participants of the test group exposed a degree of neuroticism below average. The average time was 
$2.713 \mathrm{sec}$, and the average count of views in the AOI was 9.67. The four individuals with an average score of neuroticism had $1.413 \mathrm{sec}$, and 14 views in the AOI. 1 participant was above average and performed with a time of 5,48 sec and 15 views in the AOI. Figure 2 shows a positive relation between neuroticism and the number of views. At the same time, no relation between neuroticism and time occurs. A tendency arises since the participant with the highest value of neuroticism had the longest time in the AOI. Overall, H1a: Neuroticism is positively related to the appearance of technostress is partially supported.

Extraversion: The average time and the average number of views in the AOI were measured to test H1b. Within the test group, six participants showed an average degree of extraversion. They had an average time in the AOI of $3.084 \mathrm{sec}$ and an average number of 16 views. Two participants had an above-average value of extraversion. The average time in the AOI was $0.381 \mathrm{sec}$, with an average number of two views. Based on this evidence, the higher the degree of extraversion, the lower the degree of distraction, as shown in Figure 3. Thus, H1b: Extraversion is negatively related to the appearance of technostress is supported.

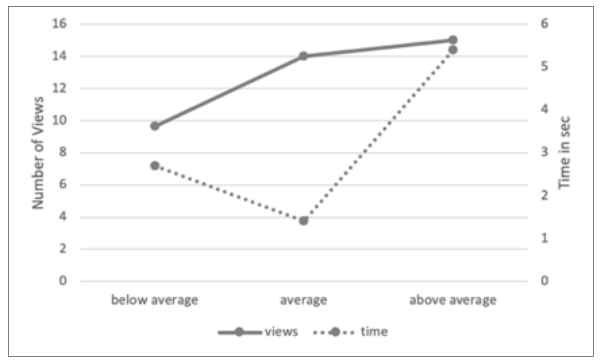

Figure 3. Results of Neuroticism

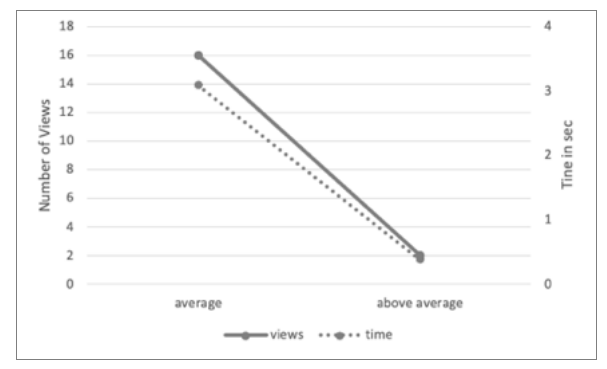

Figure 4. Results of Extraversion

Openness: The average time and the average number of views in the AOI were measured to test H1c. Two participants of the test group had a below-average degree of openness with a time of $2.611 \mathrm{sec}$ and 25.5 views. Five persons had an average personality trait with a time of $2.690 \mathrm{sec}$, and 9.6 views in the AOI. 1 individual had an above-average score on openness, which leads to a time of $0.598 \mathrm{sec}$ and one view. Figure 4 shows, in terms of views, the higher the degree of openness, the lower the number of views. A negative trend is recognizable except the average value, which is slightly higher, referring to the time. Thus, H1c: Openness is negatively related to the appearance of technostress is partially supported.

Agreeableness: The average time and the average number of views in the AOI were measured to test H1d. Two test group candidates showed below-average agreeableness with a time of $0.683 \mathrm{sec}$ and a count of 2.5 views in the AOI. Four participants showed an average score on agreeableness. Therefore, the average time of $4.285 \mathrm{sec}$ and 22.75 views were examined. Two individuals were above-average, with a time of $0.381 \mathrm{sec}$ and 2.0 views. From the results in Figure 5, no trends and relations due to the hypothesis can be identified. Hence, H1d: Agreeableness is positively related to the appearance of technostress is not supported.

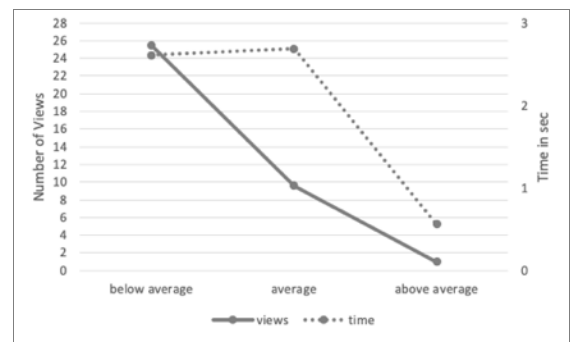

Figure 5. Results of Openness

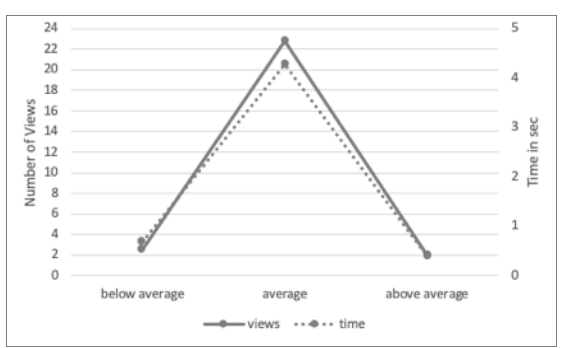

Figure 6. Results of Agreeableness

Conscientiousness: The average time and the average number of views in the AOI were measured to test H1e. Two participants showed a below-average score, with a time of $2.873 \mathrm{sec}$ and 8.5 views in the AOI. In contrast, five candidates had an average conscientiousness. They pointed time of $2.585 \mathrm{sec}$ and 16.4 views. 1 participant scores far above average in conscientiousness. Therefore, a time of $0.598 \mathrm{sec}$ and 1.0 views in the AIO was measured. As presented in Figure 6, the higher the conscientiousness, the lower time in the AOI. In terms of the number of views, no trend is recognizable. Thus, H1e: Conscientiousness is negatively related to the appearance of technostress is partially supported. The Appendix provide an overview of all results of the participants' personality. 


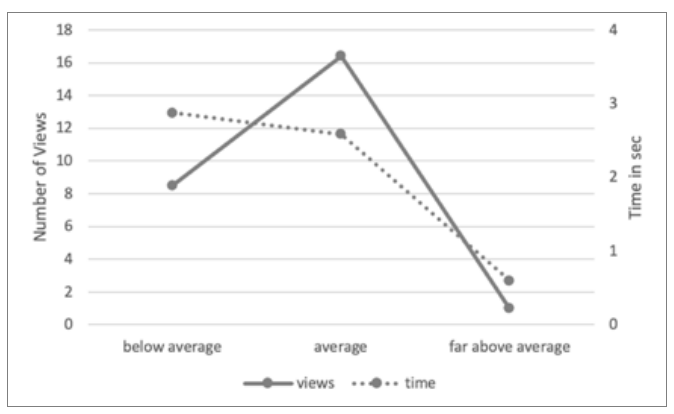

Figure 7. Results of Conscientiousness

\subsubsection{Relationship between Technical Affinity and the Appearance of Technostress}

In order to measure the degree of technical affinity, the mean-value for each participant was formed. The general mean-value $\mathrm{M}=3$ represents a neutral technical affinity. Individuals with a value below tend to have a lower affinity, whereas individuals with a score above tend to have a higher affinity. Table 3 shows the results of technical affinity.

Table 3. Results of Task Performance

\begin{tabular}{cccccc}
\hline Measure & Overall Time (min) & Overall Moves & Item & Time (min) & Moves \\
\hline Test Group & $03: 29$ & 47.75 & Technical affinity & $03: 19$ & 45.8 \\
& & & Non-technical affinity & $03: 47$ & 51 \\
\hline Control Group & \multirow{2}{*}{$03: 10$} & 47 & Technical affinity & $03: 10$ & 47 \\
& & & Non-technical affinity & - & - \\
\hline
\end{tabular}

The test group's technical affine individuals performed with an average time of 03:19 min. Moreover, it took them 45.8 moves on average to complete the task. On the opposite, the test group's non-technical affine participants needed 03:47 min and used 51 moves on average to finish the online memory. The comparison of the results presents that the test group participants with a higher technical affinity performed better than the non-technical affine ones. The delta is 00:28 min and 5.2 moves. All participants of the control group tend to be more technical affine. No one showed a value below average. It demonstrates that the control group finished the task 00:09 min faster than the technical affine test group. Consequently, the higher the degree of technical affinity, the lower the level of perceived technostress. Based on these findings, H2: Technical affinity is negatively related to technostress is supported.

\subsubsection{Relationship between Technostress and Task Performance}

Table 3 also compares the overall task performance of the test group and the control group. The test group members fulfilled the task at 03:29 min, and it took them 47.75 moves on average. Without technostress's influence, the control group finished the task in 03:10 min and used 47 moves on average. The delta is 00:19 min and 0.75 moves. Comparing these measurements, it is evident that technostress has an impact on task performance. Therefore, H3: Technostress is negatively related to task performance is supported.

\section{DISCUSSION}

This experiment proposes seven hypotheses to understand the relationship between different personality traits on the appearance of technostress. Also, the influence of technical affinity on the appearance of technostress has been observed. Additionally, the impact of technostress on task performance was measured. The hypotheses H1a-e aim to answer the first research question. H2 strives to explain the second one, and H3 is related to research question three. According to the research model, only three hypotheses are supported, three hypotheses are partially supported, and one hypothesis is not. It leads to the answer to the research questions. 
RQ1: "Which impact do personality traits have on the appearance of technostress?"

Past literature has revealed the impact of technostress creators and personality traits on outcomes such as job burnout and job engagement (Srivastava, et al. 2015). This study aimed to detect the relation between personality traits and technostress. The analysis of the hypotheses H1a-e determined that higher extraversion has a negative relation towards the appearance of technostress and plays a significant role. Neuroticism, openness, and conscientiousness show a tendency, but the hypotheses are only partially confirmed. Individuals with a higher score of neuroticism tend to experience technostress at a higher level. Open as well as conscientious individuals tend to perceive technostress at a lower level. The influence of agreeableness is not verified.

RQ2: "Which impact does technical affinity have on the appearance of technostress?"

Based on previous literature reviews, the impact of technical affinity was not examined within an experiment, yet. According to hypothesis $\mathrm{H} 2$, the research paper indicates the negative relation between technical affinity and technostress appearance. Surprisingly, the degree of technical affinity has a decisive influence. Therefore, the higher the degree of technical affinity, the lower the perceived technostress.

RQ3: "Which impact does technostress have on the task performance?"

Tams et al. investigated that individual technostress is negatively related to task performance (Tams, et al. 2018). This research paper came to a corresponding conclusion. Based on the findings of hypothesis $\mathrm{H} 3$, the test group performed worse than the control group in terms of task performance. In general, technostress reduces task performance.

Both previous studies and this study have shown that personality traits are partially related to technostress's appearance. These findings indicate that individual personality traits should be taken into account in the perception of technostress. Additionally, this research included the variable technical affinity as well. The result was that the higher the degree of technical affinity, the lower the technostress's perception.

\section{CONCLUSION}

This research paper contains certain limitations, so the results should be interpreted with caution. First and foremost, the small number of participants is the most significant limitation. Consequential only a few values can be assigned to many different variables and therefore, the hypotheses may only have limited validity. Future studies should implement a more significant sample to gather more comprehensive data to ensure a higher validity. Second, the position of the notifications was always on the right bottom of the screen. This may result in a habituation effect. For further research, the notifications could be projected on a random position on the screen to minimize such effects.

\section{REFERENCES}

Benzari, A. et al, 2020. The Rise of Technostress: A Literature Review from 1984 until 2018. Proceedings of the $28^{\text {th }}$ European Conference on Information Systems. An Online AIS Conference.

Bhattacherjee, A. 2012. Social Science Research: principles, methods, and practices, Book 3.Global Text Project.

Brod, C. 1984. Technostress: The Human Cost of the Computer Revolution. Basic Books, New York City, USA.

Burton-Jones, A. and Straub, D. W., 2006. Reconceptualizing system usage: An approach and empirical test Information Systems Research. Vol 17, No 3, pp. 228-246.

Cassar, A. et al, 2009. Cheating in markets: A laboratory experiment. Journal of Economic Behavior and Organization. Vol 72, Issue 1, pp. 240-259.

Chandra, S. et al, 2015. Do technostress creators influence employee innovation?. Pacific Asia Conference on Information Systems, PACIS 2015 - Proceedings. 
Costa, P. T. and McCrae, R. R., 1992. Revised NEO personality inventory (NEO-PI-R) and NEO five-factor inventory (NEO-FFI), The SAGE Handbook of Personality Theory and Assessment. Vol. 2, pp. 179-198.

Devaraj, U. S. et al, 2008. How does personality matter? Relating the five-factor model to technology acceptance and use. Information Systems Research. Vol. 19, No. 1, pp. 93-105

Graziano, W. G. and Eisenberg, N. H., 1997. Agreeableness: a dimension of personality. Handbook of personality psychology. pp. 795-824.

Karrer, K. et al, 2009. Technikaffinität erfassen - der Fragebogen TA-EG. Der Mensch im Mittelpunkt technischer Systeme. VDI Verlag GmbH, Düsseldorf, Germany, pp. 196-201.

Sarabadani, J. et al, 2018. 10 Years of Research on Technostress Creators and Inhibitors: Synthesis and Critique. Twenty-fourth Americas Conference on Information Systems. New Orleans, USA, pp. 1-10.

Schmitz, N. et al, 2001. Psychometric properties of the German version of the NEO-FFI in psychosomatic outpatients. Personality and Individual Differences. 31(5), pp.713-722.

Srivastava, S. C. et al, 2015. Technostress creators and job outcomes: Theorising the moderating influence of personality traits. Information Systems Journal, 25(4), pp. 355-401.

Tams, S. et al, 2018. Concentration, competence, confidence, and capture: An experimental study of age, interruption- based technostress, and task performance. Journal of the Association for Information Systems, 19(9), pp. 857-908.

Washburn, D. A. et al, 2007. A Species Difference in Visuospatial Working Memory: Does Language Link "What" with "Where"?. International Journal of Comparative Psychology. 20, pp. 55-64.

\section{APPENDIX}

Appendix 1. Results of NEOFFI of Test Group

\begin{tabular}{cccccc}
\hline $\begin{array}{c}\text { Participants } \\
\text { number }\end{array}$ & $\begin{array}{c}\text { Standard Value } \\
\text { Neuroticism }\end{array}$ & $\begin{array}{c}\text { Standard Value } \\
\text { Extraversion }\end{array}$ & $\begin{array}{c}\text { Standard Value } \\
\text { Openness }\end{array}$ & $\begin{array}{c}\text { Standard Value } \\
\text { Agreeableness }\end{array}$ & $\begin{array}{c}\text { Standard Value } \\
\text { Conscientiousness }\end{array}$ \\
\hline 1 & 41 & 59 & 38 & 43 & 52 \\
\hline 2 & 55 & 44 & 51 & 34 & 34 \\
\hline 5 & 62 & 45 & 49 & 58 & 38 \\
\hline 7 & 58 & 64 & 53 & 61 & 54 \\
\hline 9 & 38 & 51 & 54 & 45 & 47 \\
\hline 10 & 49 & 50 & 33 & 58 & 55 \\
\hline 11 & 34 & 64 & 64 & 69 & 80 \\
\hline 12 & 32 & 58 & 47 & 38 & 57 \\
\hline
\end{tabular}

